\title{
Weiterentwicklung zwingend
}

\section{Sonderregelungen sind bei einer ökologischen Steverreform im nationalen Vor- ausgang notwendig und sinnvoll. Allerdings weisen die in Deutschland reali- sierten Sonderregelungen gravierende und im Zeitverlauf zunehmende Nachtei- le auf. Eine Reform tut Not und ist im Rahmen der Weiterentwicklung der Öko- stever eigentlich auch noch für diesen Sommer vorgesehen. Allerdings gibt es auch bei den möglichen Alternativen keinen Königsweg.}

Z von Sonderregelungen ist es, fïr energi-

eintensive Unternehmen deutliche Nettobelastungen und damit Wettbewerbsnachteile zu vermeiden. Eine volle Ausnahme der Industrie von der Energiesteuer ist jedoch wirtschaftlich nicht erforderlich, ökologisch kontraproduktiv, verteilungspolitisch ungerecht sowie EU-rechtlich problematisch. Das Dilemma ist, dass keines der alternativen Modelle für Sonderregelungen gleichermaßen konzeptionell überzeugend und praxistauglich ist. Kritik ist wesentlich einfacher als konstruktive und umsetzbare Alternatiworschläge. Eine fundierte Debatte ist umso notwendiger und soll mit diesem Beitrag angestoßen werden. Im folgenden werden die wichtigsten Sonderregelungs-Modelle kurz skizziert und bewertet (1).

\section{Pauschale Steuerermäßigung}

Eine pauschale Steuerermäßigung auf 20 Prozent für alle gewerblichen Unternehmen haben DIW 1997 und DIW/ FiFo 1999 vorgeschlagen (2). Aufgrund der sehr großen Spanne der Energieintensitäten der Unternehmen verschiedener Branchen ist eine vollkommen undifferenzierte Steuerermäßigung nicht problemadäquat: Für hoch energieintensive Unternehmen ist eine Entlastung auf 20 Prozent nicht ausreichend, während für viele weniger energieintensive Unternehmen geringere Entlastungen ausreichend sind.

\section{Das derzeitige Modell: Zusätzli- cher Nettobelastungsausgleich}

Im Rahmen der ökologischen Steuerreform in Deutschland wurde ein System von Sonderregelungen für das Produzierende Gewerbe, Landwirtschaft, Teich- und Fischwirtschaft sowie Behindertenwerkstätten errichtet. Es besteht im Kern aus einer pauschalen Steuerermäßigung auf 20 Prozent, einem in jedem Fall voll zu versteuernden Sockelverbrauch und einem zusätzlichen
Nettobelastungsausgleich. Dessen Funktionsweise kann beispielhaft erläutert werden: Ein Unternehmen das trotz Steuerermäßigung noch 250.000 Mark Ökosteuern zahlt und über die Senkung der Sozialversicherungsbeiträge 100.000 Mark erhält, kann über den Nettobelastungsausgleich den Teil zurückbekommen, der das 1,2-fache der Entlastung übersteigt, im Beispielfall also 130.000 Mark

Dieser Ausgleich verhindert - im Gegensatz zur Steuerermäßigung allein - deutliche Nettobelastungen bei den energieintensiven Unternehmen. Die Kehrseite ist allerdings, dass die Unternehmen, die den Nettobelastungsausgleich beanspruchen können, keine Grenzbelastung durch die Energiesteuer und damit keine Anreize zur Energieeinsparung haben. Außerdem werden Unternehmen, die bei gleicher Energieintensität einen größeren Anteil sozialversicherungspflichtiger Beschäftigter haben, benachteiligt. Sie müssen sich eine größere Entlastung bei den Sozialversicherungsbeiträgen zurechnen lassen und erhalten einen entsprechend geringeren Nettobelastungsausgleich. Schließlich wird das im Ökosteuer-Gesetz für die zweite bis fünfte Stufe festgeschriebene Basisjahr

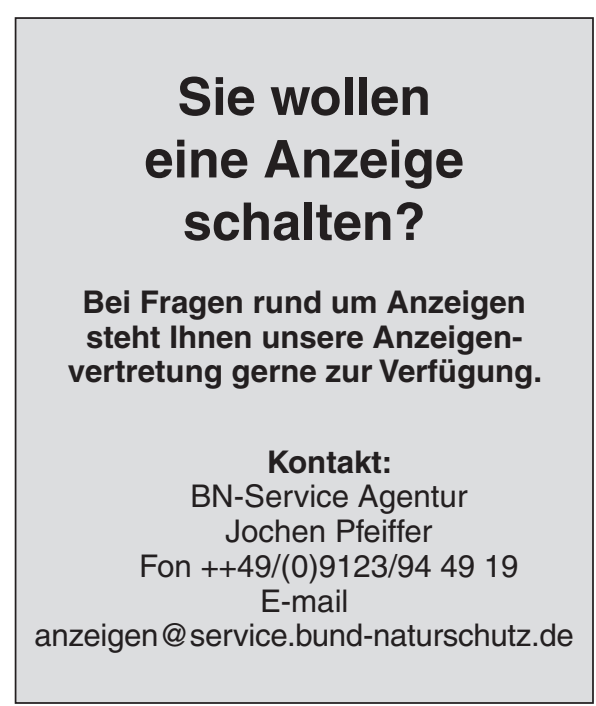

1998 für die Ermittlung der (rechnerischen) Entlastung bei der Senkung der Sozialversicherungsbeiträge im Laufe der Zeit immer realitätsferner. Für Neugründungen und bei einer Aktualisierung des Basisjahres wird im Laufe der Zeit das Problem der Umgründung gravierender: Bei Ausgründung der Beschäftigten in ein Sub-Unternehmen wird dem Unternehmen eine geringere Entlastung und damit ein höherer Nettobelastungsausgleich zugerechnet.

\section{Listenmodelle}

Hier wird eine Liste der Wirtschaftszweige erstellt, die einen ermäßigten Steuersatz - gegebenenfalls mit mehreren Ermäßigungsstufen - erhalten. Die Unternehmen werden diesen Wirtschaftszweigen zugeschlüsselt und entsprechend besteuert. Ein Listenmodell mit 26 befreiten Wirtschaftszweigen war im Gesetzentwurf der Regierungsfraktionen vom 17.11.1998 vorgesehen.

Positiv an diesem Modell ist, dass die branchenbesten Unternehmen belohnt werden. Sie erhalten Steuerermäßigungen, wie sie durchschnittlich für ihre Branche festgelegt wurden. Energieeffizientes Verhalten führt nicht zu einer Kürzung des Steuerermäßigungssatzes. Unternehmen haben keinen (kontraproduktiven) Anreiz und keine Möglichkeit, den ermäßigten Steuersatz zu beeinflussen. Dem stehen vier Nachteile gegenüber:

- Es gab eine umfangreiche wissenschaftliche und politische Debatte über die Liste der befreiten Wirtschaftszweige. Diese Debatte zeigte, dass weder der Indikator noch die verfügbaren Statistiken und Daten so belastbar waren, dass eine erhebliche Differenzierung der steuerlichen Belastung daran geknüpft werden kann.

- Aufgrund der fehlenden hinreichend harten Kriterien für die Abgrenzung der energieintensiven Wirtschaftszweige ist die Erstellung von Listen anfällig für Lobbyismus und Klageverfabren. Zahlreiche weitere Unternehmen und Verbände argumentierten, dass sie in die Liste aufgenommen werden müssten. Die Probleme vervielfältigen sich, wenn innerhalb der Liste unterschiedlich starke Steuerermäßigungen gewährt werden sollen, um hoch energieintensive Wirtschaftszweige stärker zu entlasten.

- Unter realistischen Umsetzungsbedingungen tendieren Listenmodelle zu einer Ausweitung der Liste und zu einer Gleichbehandlung der in der Liste befindlichen Wirtschaftszweige. Dementsprechend sind relativ hohe Steuermindereinnabmen zu erwarten. 
- Die Zuordnung der Unternehmen auf die Wirtschaftszweige ist bei Mehrprodukt- und bei Mehrbetriebsunternehmen hinsichtlich Verwaltungsaufwand und Manipulationsmöglichkeiten nicht unproblematisch.

\section{- Unternehmensindividuelle Energieintensitäten als Basis}

Im Kern besteht dieses Modell darin, dass bezogen auf festzulegende Schwellenwerte von Energieintensitäten (zum Beispiel bezogen auf Umsatz oder Wertschöpfung) Steuerermäßigungen gewährt werden. Der Grad der Differenzierung der Steuerermäßigung kann geeignet gewählt werden.

Durch die Kopplung der Steuerermäßigungen an den tatsächlichen Energieverbrauch bezogen auf Wertschöpfung und/oder Umsatz erhalten energieintensive und wirtschaftlich schwache Unternehmen „bedarfsgerecht“ überdurchschnittlich hohe Sonderregelungen. Ein Nachteil dieses Modells liegt in dem Anreiz, die eigenen Energiekosten über den Schwellenwert zu treiben, um die für die Sonderregelungen erforderliche Energieintensität zu erreichen. Jede Energieeinsparung verringert die zukünftigen Chancen, die Steuerermäßigung weiterhin zu bekommen. Dies ist sowohl aus Anreiz- wie auch aus Gerechtigkeitsgründen nachteilig. Auch werden energieeffiziente Pionier-Unternehmen bestraft statt honoriert: Wer in der Vergangenheit überdurchschnittlich Energie gespart hat, bekommt nun geringere Steuerermäßigungen, weil eine geringere Energieintensität vorgewiesen werden kann.

Die Zahl der Antragsfälle kann über die Energieintensitäts-Schwellenwerte sowie über einen - wie bisher - voll zu besteuernden Sockelverbrauch beeinflusst werden. Im Vergleich zu den derzeit gültigen Sonderregelungen ist der Verwaltungsaufwand eher geringer, da der Nettobelastungsausgleich entfällt und die Zahl der Antragsfälle deutlich reduziert wird.

Schließlich ist das Modell unternehmensindividueller Steuerermäßigungen nicht auf die Erstellung einer Liste von Wirtschaftszweigen, Prozessen oder Produkten angewiesen und insoweit deutlich robuster gegenüber Klageverfahren und Lobbyismus. Dies ist letztlich für die praktische Umsetzung von entscheidender Bedeutung.

\section{- Freibetragslösung}

Hier werden bezogen auf Prozesse bzw. Produkte Energiekennzahlen ermittelt und der Formulierung von spezifischen Freibeträgen zugrunde gelegt (3).
Konzeptioneller Vorteil einer Freibetragslösung ist, dass sie - anders als alle Steuerermäßigungen die Lenkungseffekte der Energiebesteuerung uneingeschränkt erhält und gleichzeitig die durchschnittliche Belastung wirksam vermindert. Weiterhin ermöglicht eine Freibetragslösung zumindest konzeptionell eine gezielte und spezifische Entlastung der hoch energieintensiven Produktionen. Damit werden auch unnötige Steuermindereinnahmen begrenzt. Dem stehen folgende Nachteile gegenüber:

- Es werden belastbare, messbare und insoweit „objektive“ Indikatoren für die Energieintensität von Prozessen benötigt, um Einwendungen und möglicherweise auch daraus folgenden Klageverfahren der nicht in der Liste befindlichen Prozesse standzuhalten.

- Für Einproduktunternehmen von standardisierten Massengütern wie Stahl oder Zement ist die Festlegung von Kennzahlen möglich. Fraglich ist jedoch, ob für alle energieintensiven Prozesse - auch in Kuppel- oder Mehrproduktunternehmen wie in der Chemischen Industrie - hinreichend homogene und messbare Outputgrößen formuliert werden können.

- Die Erarbeitung der Energiekennzahlen sowie die Formulierung der spezifischen Freibeträge bedarf weitreichender Kooperation und Konsens mit der Industrie und ist insoweit ordnungspolitisch bedenklich und anfällig für Lobbyismus.

\section{- Kopplung an ein Energie-Audit}

Mit Energie-Audits können Energiesparpotenziale mobilisiert werden, die betriebswirtschaftlich rentabel sind, jedoch aufgrund von Hemmnissen wie Informations- und Transaktionskosten nicht ausgeschöpft werden. Während für kleine Unternehmen der Aufwand eines Audits nicht lohnt und große energieintensive Unternehmen die Energiesparpotenziale aufgrund der hohen Kostenbedeutung der Energiekosten weitgehend ausschöpfen, ist ein Energie-Audit besonders sinnvoll für energieintensive mittlere sowie für weniger energieintensive größere Unternehmen.

Da der Kreis der Unternehmen, für die ein EnergieAudit sinnvoll ist, nicht deckungsgleich mit dem Kreis an Unternehmen ist, für die Sonderregelungen notwendig sind, sollten Energie-Audits nicht allein maßgeblich für die Entscheidung sein, ob und in welchem Umfang ein Unternehmen Sonderregelungen erhält. Zudem sollte aufgrund begrenzter Auditoren-Kapazitäten ein starker wirtschaftlicher Anreiz vermieden werden, dass alle Unternehmen gleichzeitig ein Energie-Audit durchführen.
Energie-Audits können also Sonderregelungen nicht ersetzen, können jedoch gut mit ihnen kombiniert werden. Beispielsweise kann in dem Modell unternehmensindividueller Steuerermäßigungen ein zusätzlicher Bonus von zwei bis fünf Prozentpunkten Steuerermäßigung für die Durchführung eines Energie-Audits gewährt werden.

\section{Fazit}

Die in Deutschland realisierten Sonderregelungen sollten mittelfristig nicht aufrecht erhalten werden und daher durch ein anderes Modell von Sonderregelungen ersetzt werden. Freibetragslösung und Listenmodelle sind schon aufgrund der Probleme der Politisierung und Beklagung der jeweiligen Liste der energieintensiven Wirtschaftszweige bzw. Prozesse oder Produkte wenig geeignet. Insbesondere eine Freibetragslösung dürfte zudem aufgrund des qualitativ und quantitativ sehr anspruchsvollen und hohen Informations- und Kooperationsbedarfs praktisch kaum umsetzbar sein.

Damit bleiben als am wenigsten nachteilige Reformoption nur unternehmensindividuelle Steuerermäßigungen. Dieses Modell hat zwar ebenfalls einige Nachteile, sie erscheinen jedoch weniger gravierend und bedeutend als die Nachteile aller alternativen Modelle.

\section{Anmerkungen}

(1) Für eine ausführliche Darstellung und Bewertung siehe die bei der Verfasserin erhältliche Langfassung.

(2) Vgl. Stefan Bach et al.: Sonderregelungen zur Vermeidung von unerwünschten Wettbewerbsnachteilen bei energieintensiven Produktionsbereichen im Rahmen einer Energiebesteuerung mit Kompensation. Berlin 1998; sowie Deutsches Institut für Wirtschaftsforschung, Finanzwissenschaftliches Forschungsinstitut an der Universitüt zu Köln: Anforderungen und Anknüpfungspunkte für eine Reform des Steuersystems unter ökologischen Aspekten, Berichte des Umweltbundesamtes Nr. 3/99, Berlin 1999.

(3) Eine Freibetragslösung wird zum Beispiel vom Finanzwissenschaftlichen Forschungsinstitut an der Universität zu Köln favorisiert.

\section{Die Autorin}

Bettina Meyer arbeitet als Referentin für Klimaschutz sowie steuerliche und wirtschaftliche Fragen des Umweltschutzes im Umweltministerium Schleswig-Holstein. Konfakt: Mercatorstr. 1-3, 24106 Kiel, Tel. 0431/ 988-7217, Fax - 7239,

E-Mail: bettina.meyer@umin.landsh.de 
(c) 20I0 Authors; licensee IÖW and oekom verlag. This is an article distributed under the terms of the Creative Commons Attribution Non-Commercial No Derivates License (http://creativecommons.org/licenses/by-nc-nd/3.o/), which permits unrestricted use, distribution, and reproduction in any medium, provided the original work is properly cited. 